\title{
The intracellular domain of the low affinity p75 nerve growth factor receptor is a death effector domain
}

\author{
HYUN HO PARK \\ Department of Biochemistry, School of Biotechnology, Yeungnam University, Gyungsan 712-749, Korea
}

Received March 9, 2009; Accepted May 20, 2009

DOI: 10.3892/mmr_00000133

\begin{abstract}
The death domain superfamily, comprising the death domain, death effector domain, caspase recruitment domain and pyrin domain subfamilies, is one of the largest classes of protein interaction modules, and plays a particularly critical function in the assembly and activation of apoptotic and inflammatory complexes. Members of the death domain superfamily share a common structural feature, the 6-helical bundle fold. However, individual subfamilies exhibit distinct structural and sequence characteristics. The most distinct feature identified in structural studies is that only the death effector domain contains a charge triad, which is formed by the E/D-RxDL motif. However, using sequence alignment and structural comparison, in the present study we found that the p75-NGFR death domain also contains a charge triad. We therefore suggest that the p75-NGFR death domain should be classified as belonging to the death effector domain.
\end{abstract}

\section{Introduction}

The low-affinity nerve growth factor receptor p75-NGFR is a well known receptor for neurotrophins. Neurotrophins are growth factors that stimulate neuronal cells to survive and differentiate, and are critical for the development and maintenance of the vertebrate nervous system (1-4). p75-NGFR is also a member of a representative subgroup of the tumor necrosis factor receptor superfamily, and can lead to apoptosis and modulate cell survival via a conserved cytoplasmic protein interaction module known as the death domain $(5,6)$. This domain, along with the death effector domain (DED), the caspase recruitment domain (CARD) and the pyrin domain (PYD), forms a death domain superfamily, one of the largest protein superfamilies (7-10). Members of its individual subfamilies mediate protein-protein interactions and regulate the assembly and activation of proteins involved in apoptosis and inflammation (10,11). Almost all oligomeric signaling com-

Correspondence to: Dr Hyun Ho Park, Departmentof Biotechnology, Yeungnam University, Gyungsan 712-749, Korea

E-mail: hyunho@ynu.ac.kr

Keywords: p75-NGFR, death domain, death effectordomain, caspase recruitment domain, pyrin domain, apoptosis, inflammation plexes in apoptosis and inflammation contain domains of the death domain superfamily. Members of the death domain superfamily exhibit a similar molecular structure, a 6-helical bundle fold with Greek key topology and an internal pseudo 2 -fold symmetry. However, variations exist in terms of the direction and length of the helices and surface properties. These differences are known to affect specificity; typically, only members of the same subfamily may interact.

Genome analysis has identified 32 death domains, 7 DEDs, 28 CARDs and 19 PYDs in the human genome (7). Death domain-containing proteins such as FADD (12), RIP (13) and TRADD (14) have been identified by the yeast two-hybrid system, while p75-NGFR (15), the ankyrins $(15,16)$, Pelle (17), Tube (17) and Myd88 (17) have been identified by sequence alignment techniques. Studies have shown that the p75-NGFR death domain differs from other death domains. First, its structure is significantly different compared to that of other death domains, such as the Fas death domain (18). Second, unlike other death domains, which have the tendency to aggregate by self-association, the p75-NGFR death domain does not selfassociate in solution (17). Finally, the p75-NGFR interaction protein does not appear to contain a death domain (19).

Although the topology of DEDs is similar to the conserved 6-helical bundle fold of the death domain superfamily, recent studies indicate a distinct conserved surface feature on DEDs, distinguishing them from other members of the death domain superfamily. This feature is a conserved hydrogen-bonded charge triad revealed by the high resolution structure of MC159 $(10,20)$. The charge triad is highly conserved in most single and tandem DEDs and is not present in other members of the death domain superfamily, suggesting that it is a characteristic feature of DEDs alone.

However, using sequence alignment, we found that the p75-NGFR death domain also contains a charge triad, and that this charge triad is located at the same position as that of the DEDs. We therefore suggest that the p75-NGFR death domain should be classified as a DED.

\section{Materials and methods}

Comparison of protein structure. The DALI protein structure comparison databases (http://ekhidna.biocenter.helsinki.fi/dali_ server), for the comparison of protein structures in $3 \mathrm{D}$, were utilized for the comparative analysis of the structure of the p75-NGFR death domain. The solution structure of the p75NGFR death domain (PDB:1NGR) was submitted (21). 
A.

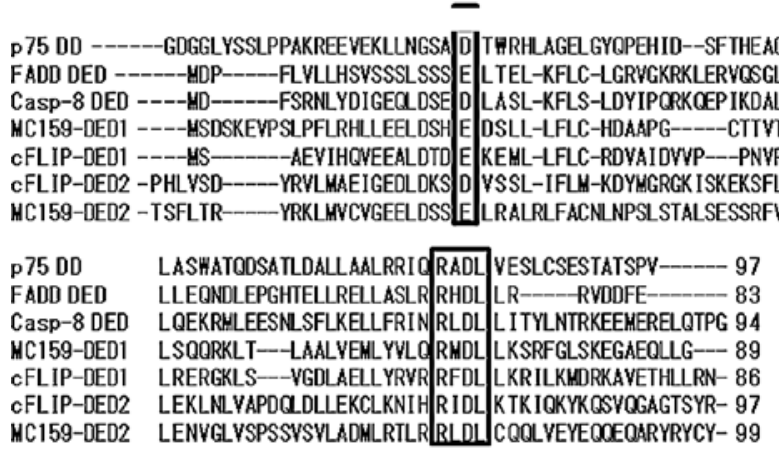

B.

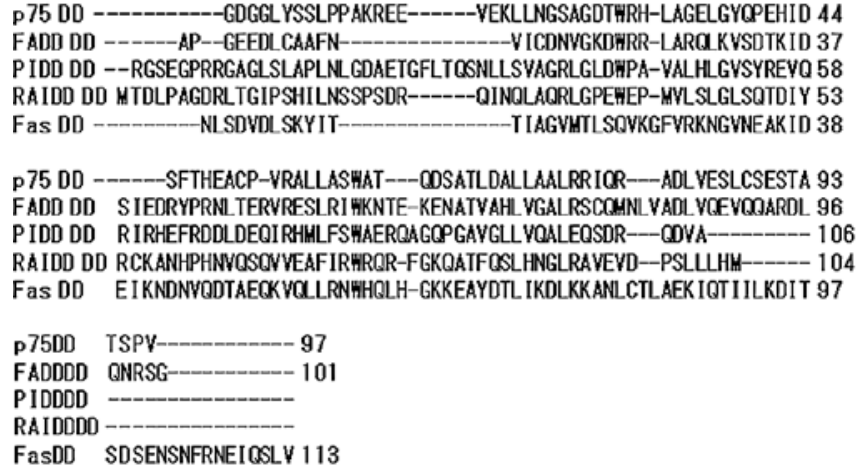

Figure 1. Sequence alignment of various death effector domains (DEDs) (A) and death domains (DDs) (B) with the p75-NGFR death domain. Alignments were performed using ClustalW. The conserved E/D-RxDL motif is outlined.
Table I. Structural similarity calculation using DALI.

\begin{tabular}{llrrrr}
\hline & PDB ID & Z-score & RMSD & LALI & LSEQ \\
\hline p75-NGFR DD & 1NGR & 21.8 & 0.0 & 85 & 85 \\
IRAK4DD & 1WH4-A & 10.6 & 1.7 & 83 & 127 \\
Pelle DD & 1D2Z-A & 10.6 & 2.1 & 84 & 102 \\
TNFR1 DD & 1ICH & 8.5 & 2.2 & 76 & 87 \\
Apaf1 CARD & 3YGS & 8.3 & 2.8 & 77 & 92 \\
Iceberg CARD & 1DGN & 7.7 & 3.0 & 78 & 89 \\
FADD DED & 1A1Z & 7.4 & 2.7 & 76 & 83 \\
Fas DD & 1DDF & 6.0 & 2.6 & 78 & 127 \\
FADD DD & 1FAD & 5.8 & 3.1 & 79 & 95 \\
\hline
\end{tabular}

Z-score, strength of structural similarity in standard deviations (only matches above a threshold of $\mathrm{Z}=5$ are reported); RMSD, positional root mean square deviation of superimposed $\mathrm{C} \alpha$ atoms in angstroms; LALI, total number of equivalanced residues; LSEQ, length of the entire chain of the equivalences structure.

Sequence alignment. The amino acid sequences of each death domain were analyzed using ClustalW (http://www.ebi.ac.uk/ Tools/clustalw2/index.html) (22).

\section{Results and Discussion}

DALI reveals that p75-NGFR shares as many similarities with the DED as it does with the death domain. Due to its importance in apoptosis and inflammation signaling, the death domain superfamily has been extensively investigated by means of biochemical and structural studies. Notably, these
A.

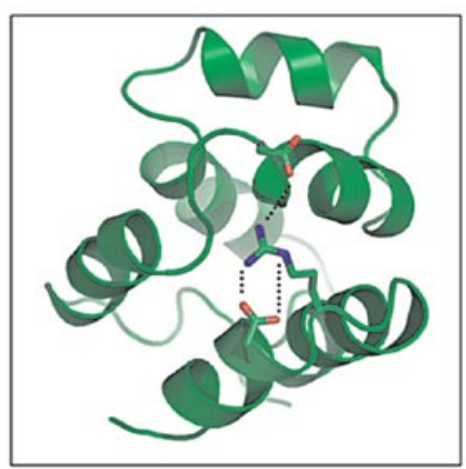

C.

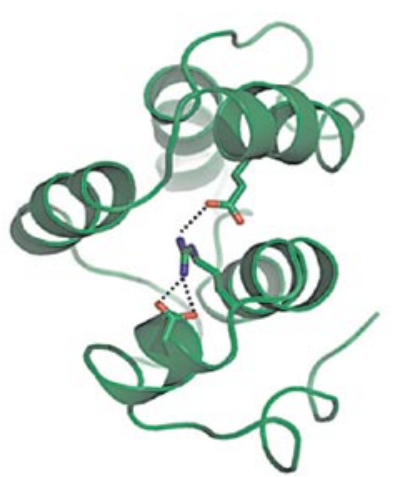

B.

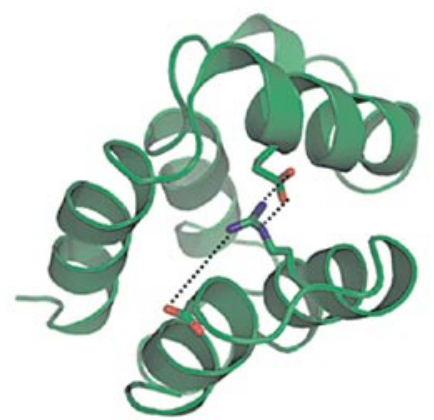

D.

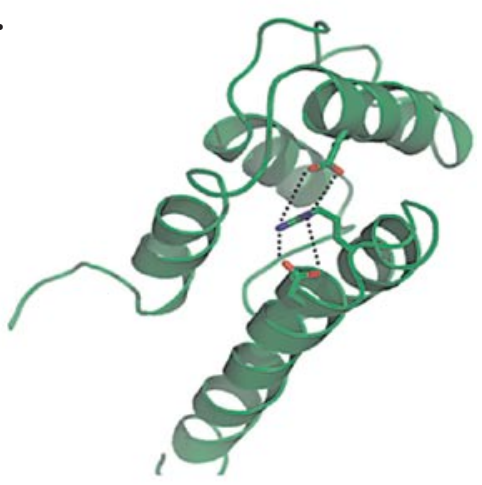

Figure 2. Conserved E/D-RxDL motif on the structure and massive H-bond. (A) p75-NGFR DD. (B) FADD DED, (C) MC159 DED1, (D) MC159 DED2. 
studies have revealed that almost all known protein-protein interactions in the superfamily are self-association or homotypic interactions with other members of the same subfamily, though the subfamilies of the death domain share a unifying feature in the form of a 6-helical bundle structural fold. This feature was first revealed by NMR spectroscopy in the Fas death domain (23), FADD DED (24), RAIDD CARD (25) and NALP1 PYD (26), classified by sequence alignment.

In the course of structural studies on the death domain and its complex, we found that the characteristics of the p75-NGFR death domain differ from those of other death domains. Using the DALI databases, 31 candidates were identified possessing a z-score above 2.0 (z-score $>3.0, n=17$; z-score $>5, n=6$ ) (Table I). Based on structural comparison, we realized that the structure of the p75-NGFR death domain most resembles that of the IRAK4 and Pelle death domains. Notably, however, subfamilies such as Afap1 CARD and FADD DED ranked in the top 4 or 6 with a z-score of 8.3 and 7.4, respectively (Table I). This indicates that the structure of the p75-NGFR death domain is as similar to the DED and CARD as it is to the death domain.

p75-NGFR contains the charge triad sequence observed in $D E D s$. Although all members of the death domain superfamily have a conserved structural fold, individual subfamilies exhibit distinct structural and sequence characteristics not shared by other subfamilies. Though the topology of DEDs is similar to the conserved 6-helical bundle fold of the death domain superfamily, recent studies indicate that there is a distinct conserved surface feature on DEDs, distinguishing them from other members of the death domain superfamily. This feature is a conserved hydrogen-bonded charge triad revealed by the high resolution structure of MC159 (Fig. 1) (10,20). The charge triad is formed by the E/D-RxDL motif and involves the Arg and Asp residues in the RxDL motif in helix H6 and the preceding loop, and an acidic residue in helix H2 (Figs. 1 and 2) (20). Extensive hydrogen bonding interactions are observed among the charged side chains with the Arg residue situated in between the two acidic residues (Fig. 2). These hydrogen bonds likely help to maintain a precise organization of the side chains, which may be functionally important. It is also possible that they play a local structural role in maintaining the conformation of this region of the DEDs. The charge triad is highly conserved in most single and tandem DEDs. Based on sequence alignment, this motif is not present in other members of the death domain superfamily, suggesting that it is a characteristic feature of DEDs alone (Fig. 1). However, using sequence alignment, we found that the p75-NGFR death domain contains a charge triad located at the same position as that of other DEDs (Figs. 1 and 2). Additionally, DED2 was found to be quite similar to the death domain of p75-NGFR (18), suggesting that p75-NGFR may be more DED-like than death domainlike. As a result, we suggest that the p75-NGFR death domain should be classified as a DED.

\section{Acknowledgements}

This study was supported by a Yeungnam University research grant in 2008, and by a grant of the Korea Healthcare Technology R\&D project, Ministry of Health, Welfare and Family Affairs, Republic of Korea (A084512).

\section{References}

1. Ibáñez C: Jekyll-Hyde neurotrophins: the story of proNGF. Trends Neurosci 25: 284-286, 2002.

2. Buxser S, Puma P and Johnson G: Properties of the nerve growth factor receptor. Relationship between receptor structure and affinity. J Biol Chem 260: 1917-1926, 1985.

3. Bothwell M: Functional interactions of neurotrophins and neurotrophin receptors. Annu Rev Neurosci 18: 223-253, 1995.

4. Bibel M and Barde YA: Neurotrophins: key regulators of cell fate and cell shape in the vertebrate nervous system. Genes Dev 14: 2919-2937, 2000

5. Huang EJ and Reichardt LF: Trk receptors: roles in neuronal signal transduction. Annu Rev Biochem 72: 609-642, 2003.

6. Rabizadeh S and Bredesen DE: Ten years on: medication of cell death by the common neurotrophin receptor p75(NTR). Cytokine Growth Factor Rev 14: 225-239, 2003.

7. Reed JC, Doctor KS and Godzik A: The domains of apoptosis: a genomics perspective. Sci STKE 239: re9, 2004.

8. Yan $\mathrm{N}$ and Shi Y: Mechanisms of apoptosis through structural biology. Annu Rev Cell Dev Biol 21: 35-56, 2005.

9. McEntyre JR and Gibson TJ: Patterns and clusters within the PSM column in TiBS. Trends Biochem. Sci 29: 627-633, 2004.

10. Park HH, Lo YC, Lin SC, Wang L, Yang JK and Wu H: The death domain superfamily in intracellular signaling of apoptosis and inflammation. Annu Rev Immunol 25: 561-586, 2007.

11. Park HH, Logette M, Raunser S, Cuenin S, Walz T, Tschopp J, and Wu H: Death domain assembly mechanism revealed by crystal structure of the oligomeric PIDDosome core complex. Cell 128: 533-546, 2007.

12. Chinnaiyan AM, O'Rourke K, Tewari M and Dixit VM: FADD, a novel death domain-containing protein, interacts with the death domain of Fas and initiates apoptosis. Cell 81: 505-512, 1995.

13. Stanger BZ, Leder P, Lee TH, Kim E and Seed B: RIP: a novel protein containing a death domain that interacts with Fas/APO-1 (CD95) in yeast and causes cell death. Cell 81: 513-523, 1995.

14. Hsu H, Xiong J and Goeddel DV: The TNF receptor 1-associated protein TRADD signals cell death and NF-kappa B activation. Cell 81: 495-504, 1995.

15. Boldin MP, Varfolomeev EE, Pancer Z, Mett IL, Camonis JH and Wallach DA: A novel protein that interacts with the death domain of Fas/APO1 contains a sequence motif related to the death domain. J Biol Chem 270: 7795-7798, 1995.

16. Cleveland JL and Ihle JN: Contenders in FasL/TNF death signaling. Cell 81: 479-482, 1995.

17. Feinstein E, Kimchi A, Wallach D, Boldin M and Varfolomeev E: The death domain: a module shared by proteins with diverse cellular functions. Trends Biochem Sci 20: 342-344, 1995.

18. Liepinsh E, Ilag LL, Otting G and Ibáñez CF: NMR structure of the death domain of the p75 neurotrophin receptor. EMBO J 16: 4999-5005, 1997.

19. Carter BD, Kaltschmidt C, Kaltschmidt B, Offenhäuser N, BöhmMatthaei R, Baeuerle PA and Barde YA: Selective activation of NF-kappa B by nerve growth factor through the neurotrophin receptor p75. Science 272: 542-545, 1996.

20. Yang JK, Wang L, Zheng L, Wan F, Ahmed M, Lenardo MJ and Wu H: Crystal structure of MC159 reveals molecular mechanism of DISC assembly and FLIP inhibition. Mol Cell 20: 939-949, 2005.

21. Holm L, Kaariainen S, Rosenstrom P and Schenkel A: Searching protein structure databases with DaliLite v3. Bioinformatics 24: 2780-2781, 2008

22. Larkin MA, Blackshields G, Brown NP, et al: Clustal W and Clustal X version 2. Bioinformatics 23: 2947-2948, 2007.

23. Huang B, Eberstadt M, Olejniczak ET, Meadows RP and Fesik SW: NMR structure and mutagenesis of the Fas (APO-1/ CD95) death domain. Nature 384: 638-641, 1996.

24. Eberstadt M, Huang B, Chen Z, Meadows RP, Ng S-C, Zheng L, and Fesik SW: NMR structure and mutagenesis of the FADD (Mort1) death-effector domain. Nature 392: 941-945, 1998.

25. Chou JJ, Matsuo H, Duan H and Wagner G: Solution structure of the RAIDD CARD and model for CARD/CARD interaction in caspase-2 and caspase-9 recruitment. Cell 94: 171-180, 1998.

26. Hiller S, Kohl A, Fiorito F, Herrmann T, Wider G, Tschopp J, Grutter MG and Wuthrich K: NMR structure of the apoptosisand inflammation-related NALP1 pyrin domain. Structure 11: 1199-1205, 2003. 